\title{
Circadian tuning with metameric white light: Visual and non-visual aspects
}

Tamara Aderneuer ${ }^{\mathrm{a}}$, Oliver Stefani ${ }^{\mathrm{b}}$, Oscar Fernández ${ }^{\mathrm{a}}$, Christian Cajochen ${ }^{\mathrm{b}, \mathrm{c}}$ and Rolando Ferrini ${ }^{\mathrm{a}}$

a CSEM Center Muttenz, Muttenz, Switzerland

b Centre for Chronobiology, University of Basel, Switzerland

c Transfaculty Research Platform Molecular and Cognitive Neurosciences (MCN), University of Basel, Switzerland

\begin{abstract}
The growing awareness of the biological effects of artificial light on humans has stimulated ample research. It is now widely accepted that asynchrony between artificial and natural light-dark cycles can elicit severe detrimental health effects. Accordingly, new research has been devoted to lighting solutions that dynamically change their color to mimic spectral changes of daylight and to account for human needs. However, in some situations, the visual properties of light must be preserved: For example professional TV video editors and shift workers who must work under standardized lighting conditions to do color correction in post-production. We have investigated the possibility to tune circadian effects using white lights that are spectrally different but nonetheless have similar color coordinates thus appear as a similar white tone. Our simulation results indicate that it is possible to modulate circadian light effects by combining LEDs for neutral white $(4000 \mathrm{~K})$, a widely used white tone for indoor lighting in europe. The results also show that the solutions combining single-color LEDs do however not meet the quality criteria from the visual point of view because their color rendering ability decays to unacceptable low levels. Combining narrowband LEDs with a broadband white LED improves the color rendering quality and we show how far circadian light effects can be tuned according to common theoretical models. The aim is to reflect daylight situations with artificial lighting, thus having a high melatonin suppression in the morning and low melatonin suppression in the evening. As a consequence our aim is to show what maximum and minimum circadian effect is possible with the same set of LEDs.
\end{abstract}

\section{Introduction}

Nowadays humans spend a long time indoors, exposed to artificial light which may pose a health risk. For example, a high dose of blue light in the evening phase delay circadian rhythms and also sleep-wake cycle which together with the intensity and amount of light received over the day can negatively impact mood (e.g. winter depression) and sleep quality $[1,2,3]$. The discovery of the intrinsically photosensitive retinal ganglion cells (ipRGCs) $[4,5,6]$ triggered substantial research efforts on lighting aspects that target human health [7]. Also in the industry domain, companies started to work on user centered lighting solutions, where it is broadly promoted as Human Centric Lighting, HCL.

Light is the most important zeitgeber (i.e. synchroniser) for the human circa- 
dian timing system (for a review see [8]). In fact, light falling on ipRGCs triggers the suppression of melatonin [9, 10], a hormone involved in the regulation of circadian physiology [1], alertness [11] and cognitive performance [12]. Current research on circadian lighting focuses primarily on retinal illuminance and the short-wavelength content of light.

As the awareness of light-related circadian effects increases also the development color-tunable (LED) luminaires grows. Such products can be tuned dynamically in their CCT to mimic natural light dynamics across the day to which humans are normally exposed to thereby preventing light-induced shifts of our internal clock. Usually, the tuning is achieved by combining LEDs with two CCTs (warm and cool) and digitally controlling their relative light outputs, i.e., the blue-yellow proportion of the resulting light [13]. More elaborate strategies involve multiple colored LEDs [14] and color conversion phosphors [15].

Despite the importance of circadian effects, lighting solutions that fail to deliver good visual experience will hardly be accepted by lighting designers, facility managers and end users in general. There are several Figures-of-Merit, FoM which are well established to describe visual performance [16]; the ones relevant for this work are presented in Table 1.

From a visual point of view, different applications demand different light characteristics. For example, in shop-retail applications the displayed products must have an appearance that closely matches that under natural light, i.e. high color fidelity. Office lighting designers however take special care to ensure comfort and productivity of the occupants and pursue uniform horizontal and non-glaring vertical illuminance levels and, although excellent color rendition is not a must, a minimum of 80 must be certified [17].

Despite the potential benefits of dynamic color tuning, white tone constancy is sometimes desirable and convenient. For example, when it is desirable to achieve certain psychological and behavioural effects through illumination with warm (reddish, low CCT) or cool light (bluish, high CCT). One effect for instance is the promotion of collaborative interaction under dim warm light [18]. The chromaticity coordinate of the illuminant determines the white tone of spectrally selective objects and due to the chromatic adaptation [19, p.71] low CCT light sources appear yellower whereas high CCT light sources appear bluer than neutral white $(\sim 4000 \mathrm{~K})[20$, p.48]. The white tone of the ambient light also influences our perception of illuminated places with high CCT values resulting in a more spacious appearance and whereas warmer lights create smaller and more familiar atmospheres [21].

Spectrally different white light sources can nonetheless be perceived as the same white tone. Such sources are called metamers and it is caused by cones' univariant response and the overlap of the cones response sensitivities. Metamerism has been investigated and patented for display applications $[22,23]$. However, display systems do not need good color rendition because they are not used to illuminate objets as it is the case for lighting systems. Selectively tuning a polychromatic white light spectrum, without changes in illuminance or color temperature can significantly influence melatonin suppression but not alertness [24]. In the study of Souman et al.,2018, three lighting conditions (Dim, high melanopic, low melanopic), each with a CCT of $2600 \mathrm{~K}$ were 
Table 1. Most relevant magnitudes and FoM used to describe light quality of light sources for visual and circadian applications.

\begin{tabular}{lll}
\hline Quantity & Abbrev. & Unit \\
\hline Vision performance & & \\
Color coordinates & $\mathrm{CIE} \mathrm{x,y}$ & 1 \\
Correlated color temperature & $\mathrm{CCT}$ & $\mathrm{K}$ \\
Luminous efficacy of radiation & $\mathrm{LER}$ & $\mathrm{lm} / \mathrm{W}$ \\
Color rendering index & $\mathrm{CRI}\left(\mathrm{R}_{\mathrm{a}}\right)$ & 1 \\
TM-30-18 & $\mathrm{R}_{\mathrm{f}}, \mathrm{R}_{\mathrm{g}}$ & 1 \\
Illuminance & $\mathrm{E}_{\mathrm{V}}$ & $\mathrm{lx}$ \\
\hline Circadian performance & & \\
Circadian stimulus & $\mathrm{CS}$ & 1 \\
Circadian action factor & $\mathrm{CAF}$ & 1 \\
Melanopic daylight equivalent illuminance & $\mathrm{E}_{\mathrm{V} 65}, \mathrm{mel}$ & $\mathrm{lx}$ \\
$\alpha$-opic illuminance & $\mathrm{E}_{\alpha}$ & $\alpha$-opic lx \\
\hline
\end{tabular}

compared. It should be noted, that the CRI was 56.8 for low melanopic and -21.8 for high melanopic.

Here we investigated the possible range of tuning circadian measures using white metamers while maintaining a high visual quality i.e. a minimum CRI of 80 . The dynamic of daylight corresponds to a variation of maximum hormon melatonin suppression in the morning to a minimum suppression in the evening. Thus here we investigated the possible range of circadian measures of white metamers. Our aim is to analyze how far lighting solutions can be "circadianly-tuned" with similar white tone and high visual quality (color fidelity).

Previous work has shown the limitations of tuning metameric white light to aim for high or low CAF. The novelty of the present paper is to predict circadian effects with the model of circadian stimulus (CS), applying it to an exemplary set of monochromatic LEDs and a broadband white LED while tuning spectra allowing for metameric white light with high visual quality. Despite the focus on the CS we also present the results of various different $\mathrm{FoM}\left(\mathrm{CAF}, \mathrm{E}_{\mathrm{z}}, \ldots\right)$.

\section{Visual and circadian effects}

In order to determine the merits of the light produced by different LED combinations we need to invoke the basic mechanism involved in human color vision. The light reaching the retina is detected by five different photoreceptors (L-, M- and S-cones, rods and ipRGCs) with different spectral sensitivity functions. The spectral responses of the L-, M- and S-cones are combined in the photopic response function $\mathrm{V}(\lambda)[25]$ which describes the overall spectral sensitivity of human vision in well-lit (photopic) conditions. The spectral circadian response on the other hand, is determined by the spectral sensitivity of melanopsin (the photopigment in ipRGCs) $\mathrm{M}(\lambda)[26]$, which is blue-shifted compared to $\mathrm{V}(\lambda)$. Hence, special care must be set when designing lighting solutions that are attuned to both aspects.

Different models and FoM have been developed in the last years to quantify the impact of light sources on the human circadian timing system, the more prominent for circadian performance being summarized in Table 1. The circadian stimulus, CS, developed by Rea and co-workers $[27,26]$ quantifies the melatonin suppression for 1-hour exposure at different illuminance levels as a function of circadian light $\left(\mathrm{CL}_{\mathrm{A}}\right) \cdot \mathrm{CL}_{\mathrm{A}}$ is a spectrally weighted illuminance and normalized such that a value 
of 1000 corresponds to $1000 \mathrm{~lx}$ of CIE standard illuminant A. The relation between $\mathrm{CS}$ and $\mathrm{CL}_{\mathrm{A}}$ is plotted in Fig. 1 which shows that CS increases with increasing $\mathrm{CL}_{\mathrm{A}}$, from zero (no melatonin suppression) to 0.7 (70\% melatonin suppression). This model considers that rods and cones contribute differently to circadian effects and multiple biological mechanisms such as blue-yellow opponency and rod saturation under photopic conditions. A unique characteristic is the non-linearity of the model which can lead to an abrupt change in melatonin suppression for spectrally similar stimuli.

Several groups working in circadian lighting research have proposed circadian

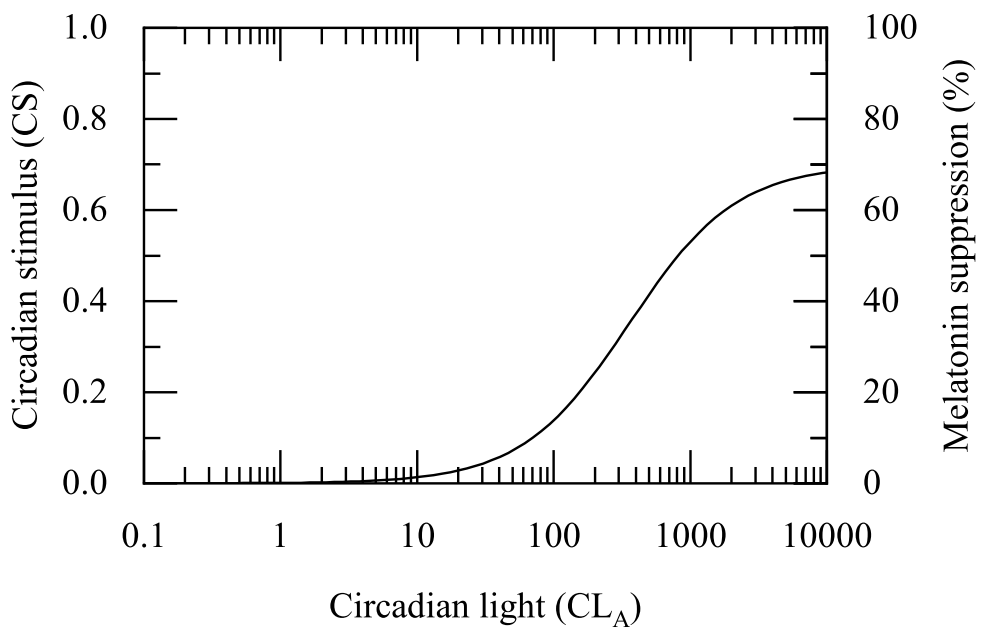

Fig. 1. The relative melatonin suppression as a function of circadian light $\left(\mathrm{CL}_{A}\right)$. It is scaled for a $2856 \mathrm{~K}$ blackbody radiation at $1000 \mathrm{~lx}$ having a value of 1000. (Formula defined in [26])

models. The Gall model [28] includes a different circadian sensitivity function $\mathrm{C}(\lambda)$ based on the experimental data of Brainard [9] and Thapan [10]. The circadian sensitivity function is implemented in the Circadian Action Factor, CAF which provides a ratio between circadian and visual merits of light sources and is easy to calculate. In the DIN SPEC 5031-100 it is named [29] melanopic action factor, with a different sensitivity curve $\mathrm{C}(\lambda)$ defined. Certain manufacturers use CAF to characterize their products[30][31]. One can thus see that one shortcoming of the $\mathrm{CAF}$ is, that for the same model different sensitivity curves are defined, which have a shifted peak in the spectrum. We have selected the $\mathrm{C}(\lambda)$ originally proposed by Gall et al. [28] [32]. Gaining acceptance and popularity is the melanopic equivalent daylight illuminance, $E_{\mathrm{V}, \text { mel }}^{\mathrm{D} 65}$, [33] which provides the (retinal) illuminance of a D65 light source with the same melanopic irradiance (also expressed in lx) as the investigated light source. Recent models by Lucas et al. and Amundadottir et al. [34] [35] consider the relative participation of different photoreceptors. Lucas model proposes the use of $\alpha$-opic illuminance, $E_{\alpha}$ where $\alpha$ stands for any of the five photopigments found in the human retina (melanopic, cyanopic, chloropic, erythropic and rhodopic illuimannce). This has found acceptance among psychologists and biologists eager for a better understanding on the mechanisms of the different photoreceptors. In this analysis, we have only considered the melanopic illuminance $E_{z}$.

It must be noted that no consensus has been reached on the most appropriate 
FoM and preferences seem to differ between biologists, psychologists, engineers and end-users since they have different perspectives and interests. For example, while end-users claim for simple evaluation criteria that facilitate the selection process, a more detailed description is often sought by researchers interested in the complete understanding of the underlying mechanisms. The authors do not favor one particular model but rather aim to provide a broad analysis.

\section{Methods}

We first combined five different narrow spectra, commonly found in commercial LEDs, and second four narrow spectra with one broadband white LED, and optimized their relative contributions for extremal (maximum and minimum) circadian effects and a preset color temperature at $4000 \mathrm{~K}$ (neutral white). We choose $4000 \mathrm{~K}$ first because this is mostly preferred for offices in Europe and the USA [36] and secondly, as previous work showed, the theoretically achievable variation of non-visual effects remain in the same range if CCT is changed between $3000 \mathrm{~K}$ and $6500 \mathrm{~K}$ [37].

An exemplary set of monochromatic LEDs is selected from OSRAM (OSRAM LED ColorCalculator; version 7.23) with the following peak wavelengths: red $(624 \mathrm{~nm})$, yellow $(594 \mathrm{~nm})$, green $(530 \mathrm{~nm})$, verde $(505 \mathrm{~nm})$ and blue $(455 \mathrm{~nm})$. In addition a conventional broadband white LED of $3600 \mathrm{~K}$ is chosen. The corresponding spectral power distributions, SPDs, were combined to produce white light of $4000 \pm 275 \mathrm{~K} \mathrm{CCT}$ and Duv $<0.006$; according to ANSI C78.377A chromaticity binning standard). The ratio of the different LEDs was changed while holding the combined power constant at $0.5 \mathrm{~W}$. In a next step, acceptable visual performance was incorporated by implementing CRI $\geq 80$ as an additional constraint. We investigated two scenarios: First a combination of five narrowband LEDs, and secondly four narrowband LEDs with one broadband white LED. Always a constant power of $0.5 \mathrm{~W}$ is kept. The irradiance was defined equal for all LED combinations to be $0.5 \mathrm{~W} / \mathrm{m}^{2}$ resulting in an illuminance in a range of 304 to $358 \mathrm{~lm}$. The spectra of all LEDs are shown in Fig. 2. The relative intensities of the LEDS were tuned to combinations resulting in a maximum and minimum circadian stimulus, CS. The algorithm was designed to find the largest circadian effect using the model proposed by Rea et al. [27], because it directly links to a biological measure that is used in studies, namely the melatonin suppression. Nevertheless the results for other common models described previously are provided as well.

\section{Results and discussion}

The results of the optimization, shown in Table 2 and Figs. 3, 4, indicate that a circadian impact holding a similiar white tone is possible, inducing a difference in melatonin suppression greater than $20 \%$. This is the case for the LED combinations of narrowband LEDs, as well as for the combination of narrowband LEDS plus white broadband LED. We will first have a look at the circadian and visual performance of the narrowband LED combinations, and in a second step what differs with the change to a narrowband plus broadband LED combination. 


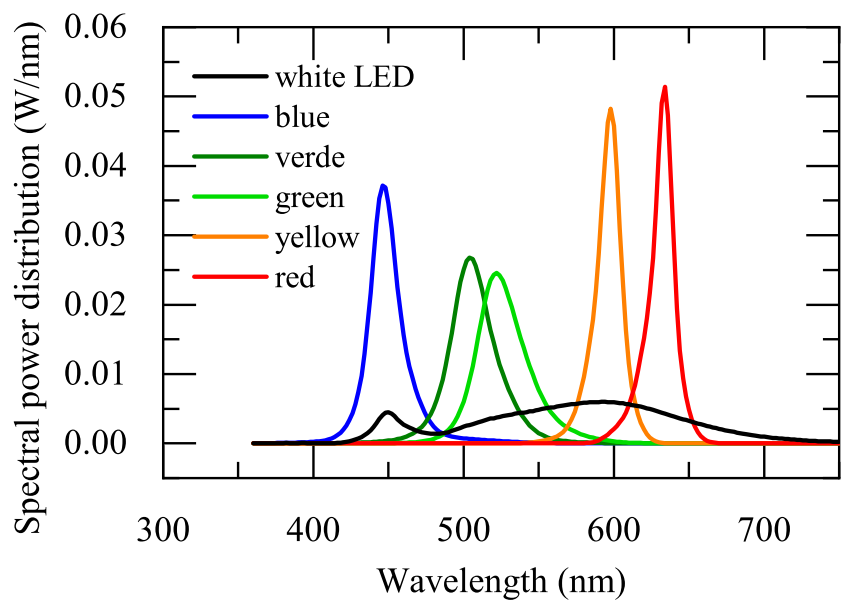

Fig. 2. Spectral power distribution of the five colored narrow-band LED (red (624nm), yellow (594nm), green $(530 \mathrm{~nm})$, verde $(505 \mathrm{~nm})$ and blue $(455 \mathrm{~nm})$ and a white LED of $3600 \mathrm{~K}$, which are combined in different ratios.

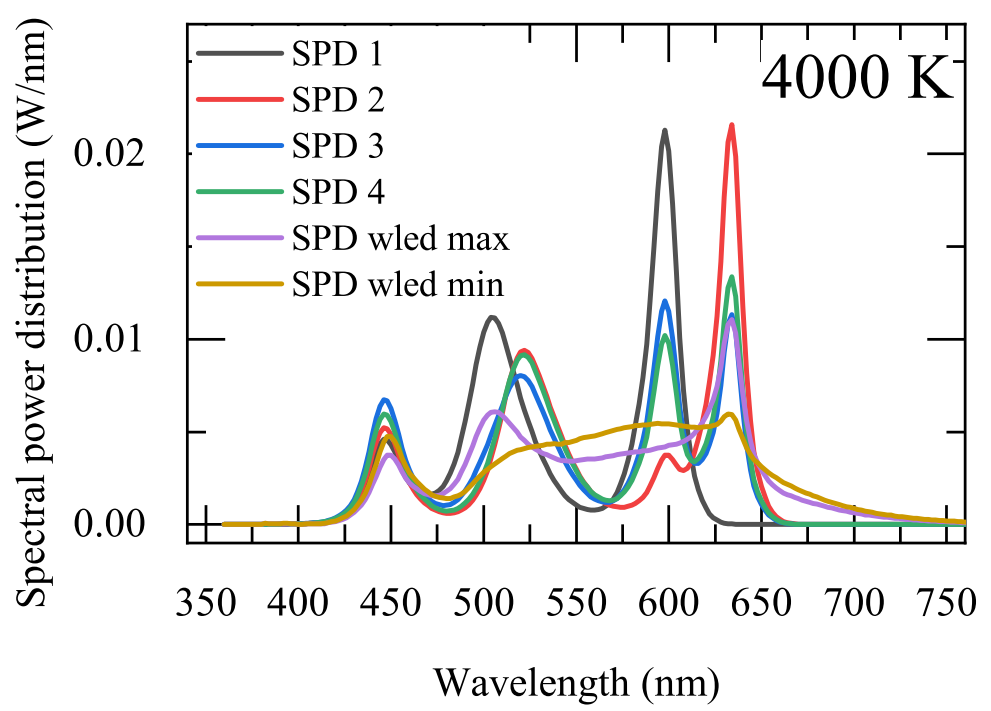

Fig. 3. Metamer LED combinations. The spectra are optimized for CS. The SPD are generated as a combination of 5 LEDs of the colors: red, yellow, green, verde, blue or of 4 colored LEDs and a broadband white LED instead of a yellow LED.All SPD resemble a CCT of $4000 \mathrm{~K}$ which is besides other standards a typical requirement for office lighting. SPD 1 is has a minimized CS, SPD 2 has a maximal CS, SPD 3 a min. CS as well as a CRI above 80, SPD 4 max. CS and CRI above 80. The SPD wled consist of a white LED instead of a yellow LED.

\section{Neutral white - 4000K CCT: narrowband LED combination}

The comparison between SDP1 compared to SPD2, optimized for maximum and minimum CS respectively, yields a $\triangle \mathrm{CS}=0.24$. In other words SPD1 has a higher melatonin suppression than SPD 2 by $24 \%$ (in the framework of Reas model). We do not want to go to more detail regarding the circadian performance before analyzing the visual performance. As reported in Table 2, SPD1 and SPD2 are characterized by CRI values of 58 and 48 respectively, well below the minimum CRI of 80 required for office lighting. 
Table 2. Analyzed parameters of the LED combinations optimized for maximal and minimal circadian performance. SPD 1 and 2 result from circadian optimization only. For SPD 3 and 4 a CRI above 80 was implemented as an additional optimization constrain. In SPD wled max and min the yellow LED is exchanged by a white LED in order to receive a better color rendering quality.

\begin{tabular}{lrrrrrr}
\hline & SPD 1 & SPD 2 & SPD 3 & SPD 4 & SPD wled max & SPD wled min \\
\hline Visual performance & & & & & & \\
CIE x & 0.384 & 0.385 & 0.378 & 0.381 & 0.396 & 0.397 \\
CIE y & 0.390 & 0.391 & 0.367 & 0.389 & 0.398 & 0.396 \\
CCT & 3995 & 3978 & 4013 & 4054 & 3755 & 3726 \\
Duv & 0.0051 & 0.0052 & -0.0039 & 0.0054 & 0.0053 & 0.0045 \\
LER & 358 & 304 & 327 & 336 & 308 & 333 \\
CRI & 58.4 & 47.8 & 80.9 & 81.0 & 85.2 & 86.5 \\
E $_{\mathrm{V}}$ & 358 & 304 & 327 & 336 & 308 & 333 \\
\hline Circadian performance & & & & & & \\
CAF & 0.54 & 0.50 & 0.55 & 0.50 & 0.55 & 0.47 \\
CL & 637 & 170 & 249 & 194 & 450 & 147 \\
CS & 0.46 & 0.22 & 0.28 & 0.24 & 0.40 & 0.19 \\
E $_{\mathrm{V}}^{\text {D65 }}$ mel & 317 & 222 & 246 & 235 & 224 & 189 \\
$\mathrm{E}_{\mathrm{z}}$ & 383 & 324 & 349 & 359 & 327 & 353 \\
\hline
\end{tabular}

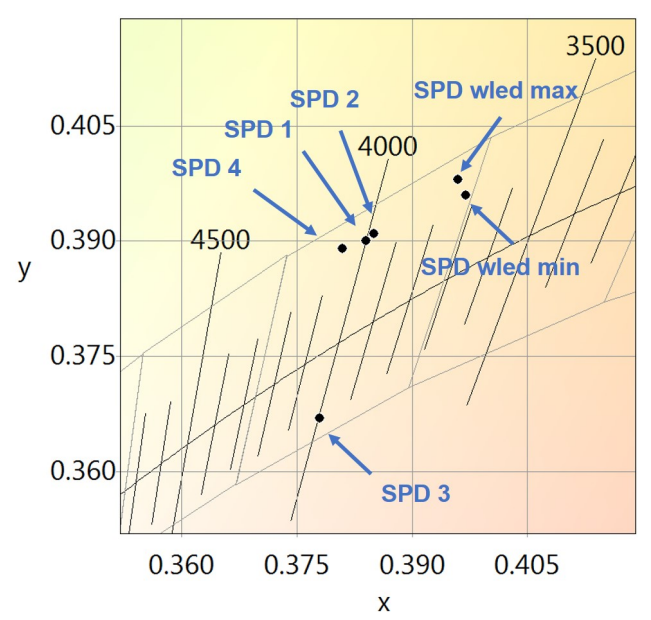

(a) CIE plot with SPDs resulting from optimization of circadian effects for CCT of 4000K

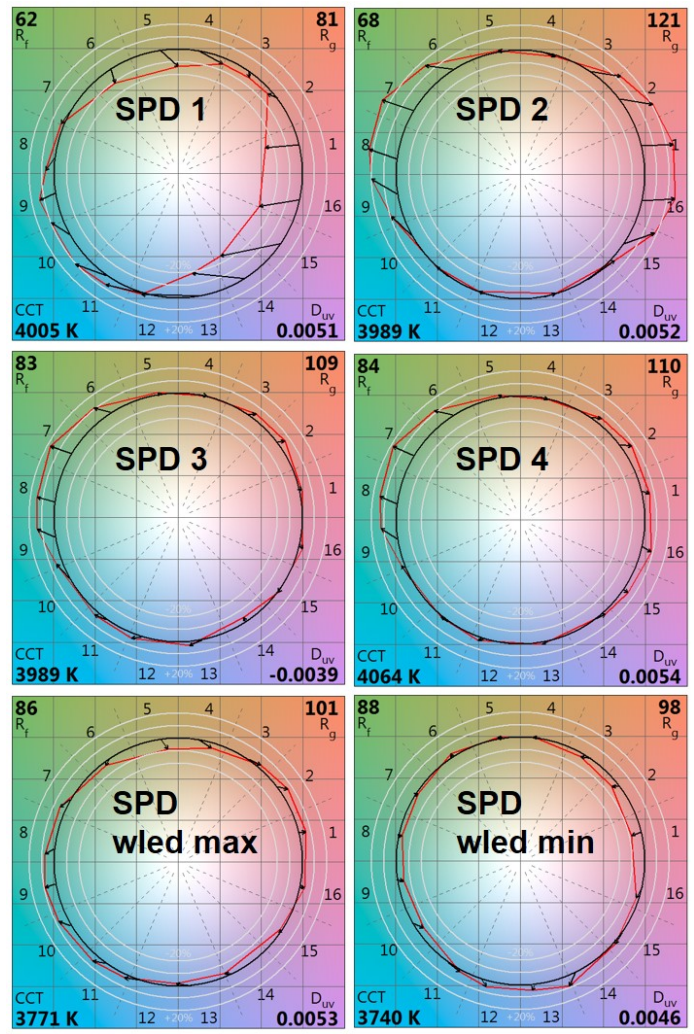

(b) TM 30-18 of the resulting four SPDs

Fig. 4. Resulting color rendering parameters CRI and TM 30-18 of the calculated SPDs. 
Therefore the optimization process was repeated this time imposing not only white point constancy (the color coordinates are given in Fig. 4(a)) but also CRI $\geq 80$. The comparison of SPD3 and SPD4 shows a small difference with $\Delta \mathrm{CS}=0.05$, i.e. a difference by $5 \%$ melatonin suppression according to the Reas model. The difference in CAF was 0.05 which corresponds to $5 \%$ difference in this model, too. As there has been no correlation to experimental findings, the interpretation of the CAF is speculative. The melanopic illuminance for SPD3 is 349 melanopic lx and for SPD4 it is 359 melanopic lx. The latter SPD was optimized to have a minimum circadian effect, such that is is surprising that the value is higher. That means the same spectrum does not go to the same direction using different circadian models. The reason for it is likely that melanopic illuminance makes a prediction on the effect on the ipRGC photoreceptors, whereas the circadian stimulus on the post processed melatonin suppression taking also the sensitivities of the other photoreceptors into account. For the melanopic daylight equivalent illuminance, SPD3 results in $\mathrm{E}_{\mathrm{V} \text {,mel }}^{\mathrm{D} 65}=246 \mathrm{~lx}$ and $\mathrm{SPD} 4$ in $\mathrm{E}_{\mathrm{V} \text {,mel }}^{\mathrm{D} 65}=235 \mathrm{~lx}$, thus the latter having a smaller value, in line with the the latter being optimized for minimum circadian effect.

Afterall, we can see that with a combination of narrowband LEDs it is not possible create a spectrum of similiar white tone that combines good visual as well as circadian performance.

\section{Neutral white - 4000K CCT: narrow- and broadband LED combination}

In a second step will look at the combination of narrowband with a broadband LED Remarkably, by exchanging a yellow LED with a broadband white LED a $\Delta \mathrm{CS}=0.2$, a maximum difference of melatonin suppression by $21 \%$, in addition to a CRI above 80 can be reached (SPD wled max and min). The difference in $\mathrm{CAF}$ was $\triangle \mathrm{CAF}=0.08$. The melanopic illuminance $\mathrm{SPD}$ wled $\max$ and $\min$ is $\Delta \mathrm{E}_{\mathrm{z}}=26$ melanopic $\mathrm{lx}$, with the SPD mimimized for circadian impact having the greater value. The the melanopic daylight equivalent illuminance differs for SPDwled $\max$ and min by $\Delta \mathrm{E}_{\mathrm{V}, \text { mel }}^{\mathrm{D} 65}=35 \mathrm{~lx}$ where the latter has the smaller value. The results are therefore analogue to the one of SPD 3 and 4.

It is known that CRI does not truly reflect the color rendition abilities of light sources [38] and several alternatives have been proposed. Therefore we want to shortly report on more detailed calculations regarding the color fidelity of our spectra. A measure that is gaining a rather good support is the TM-30-18 model [39]. which was thus calculated in addition to the CRI. The results provided conclusions in line with the ones for CRI. The fidelity index, $\mathrm{R}_{\mathrm{f}}$, of SPD3, SPD4, SPDwled max and min was significantly higher than that of SDP1 and SPD2 which also displayed a lower gamut index, $R_{g}$, indicative of the under-saturation of green tones. For details see Fig. 4(b))

In summary, the results show the limits of potential circadian tuning for 4000K when adhering to an acceptable visual level to have a minimum color fidelity. The combinations of single-color LEDs does allow a white tone of very good color rendering quality. However, simultaneously realizing proper circadian tuning it is not possible. Combinations that include a broadband white LED improve the color rendering quality and can simultaneously ensure visual and non-visual aspects: circadian tuning is possible in a larger range (melatonin suppression from $20 \%$ to $40 \%$ ) jointly with providing a sufficient color rendering quality (CRI above 80 ). 


\section{Conclusions}

Our results show the range in which non-image forming effects can be tuned by changing the spectrum while keeping the visual appearance at an acceptable quality maintaining a white tone.

Using metamers, we need to distinguish between light sources for illumination of the environment and illumination of displays. When light is used to illuminate objects in the environment, a good color experience of the user needs to be guaranteed.

With a selection of different narrowband colored LEDs it is possible to have a CS ranging from 0.22 to 0.46 , a difference of $24 \%$ in melatonin suppression, only though at the cost of an unacceptable color rendering quality. By combining narrowband colored LEDs with a broadband white LED, it is possible to overcome this drawback. If the white LED has a gap in the spectrum where the sensitivity for non-visual effects is high, we could tune melatonin suppression to the same extend (difference of $21 \%$ ) and keep an acceptable color rendering quality.

In this work one set of LEDs was selected to analyze them using the circadian stimulus as circadian model, which has not been done before. Because it is a non linear model, this hinders though to implement it in an algorithm to optimize for the best set of different LEDs. However, the selection presented here reflects other combinations as well, holding the following properties: One key is the presence of a white broadband LED to ensure color fidelity. In addition multiple colored LEDs to tune for the circadian effect, which should be spread out over the visible range. We expect though that further improvement is possible fine tuning on their selection. Our results corroborate previous theoretical analysis reporting limitations for tuning circadian effects via the spectrum of light with a constant white tone [37, p.156]. Although so-called "circadian" or "human centric" lighting is very fashionable and promoted by many LED manufacturers, metameric light is merely used for non-visual effects but only for very specific visual applications (e.g. light cabins for colometry). Adding color channels to lighting systems results in massive engineering complexities (LED precise tuning) and is therefore usually not deployed by lighting companies.

\section{Appendix}

Here we want to list the details on the circadian models and respective formulas used.

The circadian stimulus (CS) was proposed by Rea et al. [27]. It is a function of Circadian Light $C L_{A}$

CS can be expressed as:

$$
C S=0.7\left(1-\frac{1}{1+\left(\frac{C L_{A}}{355.7}\right)^{1.1026}}\right)
$$


where the circadian light is defined as

$$
C L_{A}=\left\{\begin{array}{rr}
1622\left[\int M_{\lambda} E_{\lambda} d \lambda+\left(a_{b-y}\left(\int \frac{S_{\lambda}}{m p_{\lambda}} E_{\lambda} d \lambda-k \int \frac{V_{\lambda}}{m p_{\lambda}} E_{\lambda} d \lambda\right)-\right.\right. \\
\left.\left.a_{\text {rod }}\left(1-e^{\frac{-\int V_{\lambda}^{\prime} E_{\lambda} d \lambda}{R o d s a t}}\right)\right)\right] \\
1622 \int M_{\lambda} E_{\lambda} d \lambda & \text { if } \int \frac{S_{\lambda}}{m p_{\lambda}} E_{\lambda} d \lambda-k \int \frac{V_{\lambda}}{m p_{\lambda}} E_{\lambda} d \lambda \geq 0 \\
\text { if } \int \frac{S_{\lambda}}{m p_{\lambda}} E_{\lambda} d \lambda-k \int \frac{V_{\lambda}}{m p_{\lambda}} E_{\lambda} d \lambda<0
\end{array}\right.
$$

The circadian action factor (CAF) is developed by [28] and is defined as:

$$
C A F=\frac{\int C(\lambda) E(\lambda) d \lambda}{\int V(\lambda) E(\lambda) d \lambda}
$$

The melanopic equivalent daylight illuminance $E_{\mathrm{V}, \text { mel }}^{\mathrm{D} 65}[33]$ can be expressed as:

$$
E_{\mathrm{V}, \text { mel }}^{\mathrm{D} 65}=\frac{K_{\mathrm{V}, \mathrm{mel}}}{K_{\mathrm{V}, \text { mel }}^{\mathrm{D} 65}} E_{\mathrm{V}}
$$

where $\frac{K_{\mathrm{V} \text {,mel }}}{K_{\mathrm{V} \text {,mel }}^{\mathrm{D} 65}}$ is the melanopic daylight (D65) efficacy ratio.

The the alpha-opic illuminance $\left(E_{\alpha}\right)[34]$ is described by

$$
E_{\alpha}=72983 \int E_{e}(\lambda) N_{\alpha}(\lambda) d \lambda
$$

where $E_{e}(\lambda)$ is the incident illuminance and $N_{\alpha}(\lambda)$ represents the photoreceptor spectral sensitivity and is different for each type. Therefore the authors recommend to report all types in biological studies such that comparisons can be made.

\section{References}

[1] Lockley, S. W., Brainard, G. C. \& Czeisler, C. A. High Sensitivity of the Human Circadian Melatonin Rhythm to Resetting by Short Wavelength Light. The Journal of Clinical Endocrinology 85 Metabolism 88, 4502-4505 (2003).

[2] Lewy, A. J., Kern, H. A., Rosenthal, N. E. \& Wehr, T. A. Bright artificial light treatment of a manic-depressive patient with a seasonal mood cycle. The American Journal of Psychiatry 139, 1496-1498 (1982).

[3] Lack, L. \& Wright, H. The Effect of Evening Bright Light in Delaying the Circadian Rhythms and Lengthening the Sleep of Early Morning Awakening Insomniacs. Sleep 16, 436-443 (1993).

[4] Berson, D. M., Dunn, F. A. \& Takao, M. Phototransduction by Retinal Ganglion Cells That Set the Circadian Clock. Science 295, 1070-1073 (2002).

[5] Hattar, S., Liao, H.-W., Takao, M., Berson, D. M. \& Yau, K.W. Melanopsin-containing retinal ganglion cells: Architecture, projec- 
tions, and intrinsic photosensitivity. Science 295, 1065-1070 (2002). https://science.sciencemag . org/content/295/5557/1065.full.pdf.

[6] Provencio, I., Jiang, G., De Grip, W. J., Hayes, W. P. \& Rollag, M. D. Melanopsin: An opsin in melanophores, brain, and eye. Proceedings of the National Academy of Sciences 95, 340-345 (1998). https: //www.pnas .org/content/95/1/340.full.pdf.

[7] Lighting for people (2019). URL http://lightingforpeople.eu/ human-centric-lighting/. Accessed: 2019-04-25.

[8] Cajochen, C., Chellappa, S. L. \& Schmidt, C. Circadian and Light Effects on Human SleepinessAlertness. In Garbarino, S., Nobili, L. \& Costa, G. (eds.) Sleepiness and Human Impact Assessment, 9-22 (Springer Milan, Milano, 2014).

[9] Brainard, G. C. et al. Action Spectrum for Melatonin Regulation in Humans: Evidence for a Novel Circadian Photoreceptor. Journal of Neuroscience 21, 64056412 (2001).

[10] Thapan, K., Arendt, J. \& Skene, D. J. An action spectrum for melatonin suppression: evidence for a novel nonrod, noncone photoreceptor system in humans. The Journal of Physiology 535, 261-267 (2001).

[11] Cajochen, C. Alerting effects of light. Sleep Medicine Reviews 11, 453-464 (2007).

[12] Vandewalle, G., Maquet, P. \& Dijk, D.-J. Light as a modulator of cognitive brain function. Trends in Cognitive Sciences 13, 429-438 (2009).

[13] Tridonic. Mit tunable white $\mathrm{zu}$ individualisiertem licht (2015). URL https://www.tridonic.ch/ch/products/tunable-white.asp. Accessed: 201902-25.

[14] Chew, I., Kalavally, V., Tan, C. P. \& Parkkinen, J. A Spectrally Tunable Smart LED Lighting System With Closed-Loop Control. IEEE Sensors Journal 16, 4452-4459 (2016).

[15] Fernández, O., Ferrini, R., Barruetabe, L. \& Ivanoviciai, S. Ultrathin direct-lit led module with beam-shaping thin-film optics. LED professional Review 48-54 (2017).

[16] Wyszecki, G. \& Stiles, W. S. Color Science: Concepts and Methods, Quantitative Data and Formulae (Wiley-Interscience, New York, 2000), 2. edn.

[17] Commission Regulation (EU). Commission regulation (eu) no 1194/2012 of 12 december 2012 implementing directive 2009/125/ec of the european parliament and of the council with regard to ecodesign requirements for directional lamps, light emitting diode lamps and related equipment text with eea relevance (2012).

[18] Kombeiz, O., Steidle, A. \& Dietl, E. View it in a different light: Mediated and moderated effects of dim warm light on collaborative conflict resolution. Journal of Environmental Psychology 51, 270-283 (2017).

[19] Khanh, T. Q., Bodrogi, P. \& Vinh, T. Q. The White Point of the Light Source. In Color Quality of Semiconductor and Conventional Light Sources, 71-90 (John Wiley \& Sons, Ltd, 2017).

[20] Cuttle, C. Lighting design: A perceptionbased approach. Lighting Research 6 Technology 47, 763-764 (2015).

[21] Luxreview. Does lighting boost retail sales? (2015). URL https ://luxreview .com/article/2015/06/does-lighting-boost-sales-. Accessed: 2019-02-25.

[22] Allen, A. E., Martial, F. P., Lucas, R. J., Hazelhoff, E. M. \& Cajochen, C. Exploiting metamerism to regulate the impact of a visual display on alertness and melatonin suppression independent of visual appearance. Sleep 41 (2018).

[23] Bues, M., Stefani, O. \& Pross, A. Display sys- 
tem having circadian effect on humans (2012). URL https://patents.google.com/patent/US20120069551A1/zh.

[24] Souman, J. L. et al. Spectral Tuning of White Light Allows for Strong Reduction in Melatonin Suppression without Changing Illumination Level or Color Temperature. Journal of Biological Rhythms 33, 420-431 (2018).

[25] Commission Internationale de l'Eclairage. Light as a true visual quantity: Principles of measurement | CIE (1994).

[26] Rea, M., Figueiro, M., Bierman, A. \& Hamner, R. Modelling the spectral sensitivity of the human circadian system. Lighting Research 86 Technology 44, 386-396 (2012).

[27] Rea, M. S., Figueiro, M., Bierman, A. \& Bullough, J. Circadian light. Journal of Circadian Rhythms 8 (2010).

[28] Gall, D. \& Bieske, K. Definition and measurement of circadian radiometric quantities. In Proceedings of the CIE Symposium 2004 on Light and Health: NonVisual Effects, 129-132 (Commission internationale de l'eclairage, Vienna, Austria, 2004).

[29] DIN. Optical radiation physics and illuminating engineering - Part 100: Melanopic effects of ocular light on human beings - Quantities, symbols and action spectra. DIN SPEC 5031-100:2015-08 (Beuth Verlag GmbH, Berlin, 2015).

[30] Firalux. Mit tunable white $\mathrm{zu}$ individualisiertem licht (2018). URL https://www.firalux.ch/images/katalog/Kiteo/18_08_KITEO_Katalog_EN.PDF. Accessed: 2019-04-25.

[31] Vossloh \& Schwabe. Led line csp tuneable w4 (2018). URL https://www.vossloh-schwabe.com/uploads/tx_espdf search/ LED-Line-CSP-TW_L28_56_W4_DE.pdf. Accessed: 2019-04-25.

[32] Gall, D. \& Lapuente, V. Beleuchtungsrelevante Aspekte bei der Auswahl eines frderlichen Lampenspektrums (2002).

[33] Commission Internationale de l'Eclairage. CIE system for metrology of optical radiation for iprgc-influenced responses to light (2018).

[34] Lucas, R. J. et al. Measuring and using light in the melanopsin age. Trends in Neurosciences 37, 1-9 (2014).

[35] Amundadottir, M., Lockley, S. \& Andersen, M. Unified framework to evaluate non-visual spectral effectiveness of light for human health. Lighting Research \&6 Technology 49, 673-696 (2017).

[36] Institute, N. B. \& Benya, J. Advanced Lighting Guidelines (New Buildings Institute, Incorporated, 2001).

[37] Deutsche Lichttechnische Gesellschaft e.V. LICHT 2016 : Karlsruhe, 25. - 28. September ; Tagungsband - Proceedings ; [22. Gemeinschaftstagung = 22nd Associations Meeting] (KIT Scientific Publishing, 2016).

[38] Houser, K., Mossman, M., Smet, K. \& Whitehead, L. Tutorial: Color Rendering and Its Applications in Lighting. LEUKOS 12, 7-26 (2016).

[39] Illuminating Engineering Society. IES method for evaluating light source color rendition (2018). 